\title{
Research on the Evolution Cost Control of Small-Micro Enterprise Multinational Operation
}

\author{
Li Jun \\ School of Management, Wuhan University of Technology, Wuhan 430070, China
}

\begin{abstract}
To explore small and micro enterprise's multinational management, new theory, new method and new perspective are needed. A growth path in multinational operation using niche genetic algorithm is constructed. In order to help decision makers making more scientific judgment according to the overall environment, a function of a learning exchange cost is embedded in the evolution process, which would be an important index for the feasibility analysis on multinational operation of small micro enterprise.
\end{abstract}

KEYWORD: Small and Micro Enterprises; Multinational operation; Niche; Cost

As the independent innovation ability and the comprehensive economic strength has increased, China's small and medium-sized enterprises have gradually enter the international track, also emerged a large number of Small and micro businesses. The traditional international economic theory is difficult to fully explain the emergence of small and mediumsized enterprise multinational business, such as technology localization theory [1], the small-scale technology theory[2], the dynamic comparative advantage theory [3], etc. From an evolutionary perspective, this paper evaluate the small enterprises in the internationalization tide of fitness, to explore suitable for small micro enterprise multinational pattern and the method of quantitative evaluation, and then assisted small micro enterprises to make decisions in the process of growth, gain more market and opportunities.

\section{SMALL MICRO ENTERPRISE CONNOTATION AND THE SIGNIFICANCE OF TRANSNATIONAL OPERATION}

In general, small micro enterprise is a broad concept, can be small businesses, the micro enterprises, family enterprises and individual business collectively, and the four categories are different. Through the analysis of the existing situation, the situation of the modern small micro enterprise of can be summarized as four small [4] : a small number, small amounts of money, a small amount of sales, a small amount of equipment, may according to individual companies, there are more "less". These "less" constitute the small micro enterprise of "small" and "micro", but under the circumstances of China's policy to encourage and reform, a collection of small micro enterprise is a very large collection.

At present, the small micro enterprise multinational business there is no specification and particular mode, the current is still in the exploratory stage. Enterprise institute under the state council development research center, research summarizes the 24 kinds of Chinese enterprise internationalization business model [5], but the pattern of considerations for static and qualitative analysis. There are many scholars combined with the growth of the small micro enterprise features, to explore the small micro enterprise evolution path of the index system and evaluation method [6-7], but the lack of dynamic evaluation method, makes it hard for the growth of the small micro enterprise reflect the evaluation of enterprise adaptability to the environment, In this paper, the small micro enterprise multinational business niche genetic algorithm is a new exploration of quantitative analysis of the small micro enterprises transnational operation path.

\section{SMALL MICRO ENTERPRISE MULTINATIONAL BUSINESS NICHE GENETIC ALGORITHM}

In small micro enterprise development and competitiveness of ascension is recessive goal, difficult to dynamic quantitative analysis, fusion niche technology and genetic algorithm, this paper 
puts forward the algorithm, the fitness of international operation of small micro enterprise. Individual fitness value reflects the various aspects of information and abilities, has a good fitness of individuals on behalf of the survival of the individual has the stronger ability, while poor individuals will be eliminated, making the high fitness individuals blooms, full of the whole group.

Considering the commonness of small micro enterprise multinational business and niche ideology, micro enterprises transnational operation to carry out mainly in the form of cluster and the union, so the niche technology can be used to dynamic evolution path of transnational operation for small micro enterprises. Practice has proved that most likely growth of enterprises is to adapt to the environment.

To develop cluster or alliance group, as a species, small micro enterprise is the population of individuals. Small micro enterprise multinational business usually in occasionally the export of products, technology, services as a start, or to the success of a specific enterprise as the goal to design its own transnational operation strategy. Niche there is a core of the individual, as the center, as the core enterprise in multinational management, In $\sigma$ as the radius of niche within the scope of the individual's fitness is the largest. About the niche radius set did not adopt the method of general, can use hamming distance [8], as shown in formula (1).

$$
d_{i j}=\left\|X_{i}-X_{j}\right\|=\sqrt{\sum_{k}^{M}\left(x_{i k}-x_{j k}\right)^{2}}
$$

$X_{i}$ and $X_{j}$ said individual in the population, $d_{i j}$ said the distance between the two. $M$ is the dimension of consideration of the individual. Niche radius $\sigma$ will affect the performance of the algorithm, the niche radius is bigger, is beneficial to maintain the population diversity, but the population evolution is slow, the algorithm convergence speed is also slow. Niche radius is lesser, is beneficial to improve the convergence speed of the algorithm, but lack of fast population diversity, algorithm is easy to premature and fall into local optimum. This paper set up the adaptive niche radius, before the population genetic division scope, dynamic form independent search space, dynamic changes in convergence speed and search the global optimal value.

$$
\sigma=\sigma_{0} \cdot e^{\left(-d_{a} / D_{m}\right)}
$$

Among them, $\mathrm{d}_{\mathrm{a}}$ is the average hamming distance between population, $\mathrm{D}_{\mathrm{m}}$ is the most big fellow hamming distance of populations. $\sigma_{0}$ is the setting of the initial radius.

$$
d_{a}=\sum_{i=1}^{N}\left\|X_{o}-X_{i}\right\| / N
$$

The $\mathrm{X}_{\mathrm{o}}$ as individual core, $\mathrm{N}$ said the number of all individuals in niche.

Small micro enterprise multinational management of the bottom line is to guarantee the survival of enterprises, namely the individual continues to exist in the population, the development goal is to become a core member of the population, with higher competitiveness. Fitness function as formula (4)

$$
\operatorname{fitness}\left(X_{i}\right)=\text { Fit } \cdot\left(1-d_{i} / D_{m}\right)
$$

Among them, the fitness $\left(\mathrm{X}_{\mathrm{i}}\right)$ said the fitness of the individual $X_{i} \cdot d_{i}$ is $X i$ and core individual hamming distance. fit is an iterative optimal fitness in the round. $d_{i} / D_{m}$ said that the individual from the population within the distance of the best individual and the ratio of maximum distance within populations. There are crowding mechanism in the niche technology, to punish or eliminate lower fitness of the individual. In this paper, keep the low fitness of individuals, through crossover and mutation to improve the individual. The adaptability of the enterprise is the most important is to learn communication, including the exchange of material and spiritual level, learning communication is indispensable skill for enterprises to survive in the market, in the niche technology embodied in populations of crossover and variation. And route choice model about small micro enterprises transnational operation, is reflected in the study and communication strategy.

Crossover: small micro enterprise itself the resource exchange with other enterprises, and the exchange of there is a certain probability, the choice of exchange object is also related to the overall situation, For example when a particular resource distribution is intensive or concentration, the object of exchange is very clear, on the other hand, when a scattered distribution of resources, you will need to test for many times, to find the appropriate objects of exchange.

$$
p_{c}=p_{1}+\left(p_{2}-p_{1}\right) \mathrm{e}^{\left(-H^{2}\right)}
$$

$\mathrm{p}_{\mathrm{c}}$ as the crossover probability, $\mathrm{H}$ as niche entropy, $\mathrm{p}_{1}, \mathrm{p}_{2}$ is artificial initial setup probability.

Variation: small micro enterprise according to the change of living environment, the internal reform of the enterprise itself, innovative business model.

$$
q_{c}=q_{1}+\left(q_{2}-q_{1}\right) \mathrm{e}^{\left(-H^{2}\right)}
$$

Among them, the $\mathrm{q}_{\mathrm{c}}$ is the crossover probability, $\mathrm{H}$ is the entropy niche, $\mathrm{q}_{1}$ and $\mathrm{q}_{2}$ is artificial initial setup probability.

$$
H=-\sum_{k=1}^{\theta} \frac{\operatorname{num}\left(x_{o, k}\right)}{n} \ln \frac{\operatorname{num}\left(x_{o, k}\right)}{n}
$$

$\operatorname{Num}\left(\mathrm{x}_{\mathrm{o}, \mathrm{k}}\right)$ with the individual $\mathrm{x}_{\mathrm{o}, \mathrm{k}}$ as the core, the number of individuals in $\sigma$ radius, $\theta$ as the core 
number of individuals. The entropy theory shows that when more core individuals in a population and individuals in the population are evenly distributed, the species diversity is good, the niche of entropy value is bigger. When less individual core in population and population distribution becomes concentrated in a small core of individuals, population diversity is poorer, the smaller niche entropy [9].

\section{SMALL MICRO ENTERPRISE NICHE EVOLUTION COST FUNCTION}

Niche technology, in fact, is a kind of search algorithm of optimal value in the process of evolution, to consider the cost factor in the process of evolution or search. In practice, due to the policy change and the change of market environment, often learn communication all need to pay the price or cost. When the niche of entropy value is small, because the search direction is more clear, study and communication of search costs less, when larger niche entropy, fuzzy search direction, learning communication search cost is larger. Small micro enterprises to develop more is limited by funding, financing way is less, so the cost of study and communication is an important decision factors in the process of growth. The cost of individual study and communication functions as shown in formula (8).

$$
C\left(X_{i}\right)=\left(d_{i} / D_{m}\right)\left[1-\mathrm{e}^{\left(-H^{2}\right)}\right]
$$

Individual $\mathrm{X}_{\mathrm{i}}$ learning communication costs by population distribution of the internal and external influence. When individuals in a population from which the optimal individual far, population distribution is more homogeneous, learn the communication cost is larger, the lack of play the leading role of enterprises in the population. When individuals in the population distance the best individual is relatively close, even became the best individual, population distribution is concentrated, study and communication cost is small, the entire populations have strength strong core enterprise, playing a leading role.

Based on the study and communication costs as the main decision-making elements of small micro enterprise growth, When cost is larger, the growth of the small micro enterprise is large, and when the cost is low, small micro enterprise can get better learning effect.
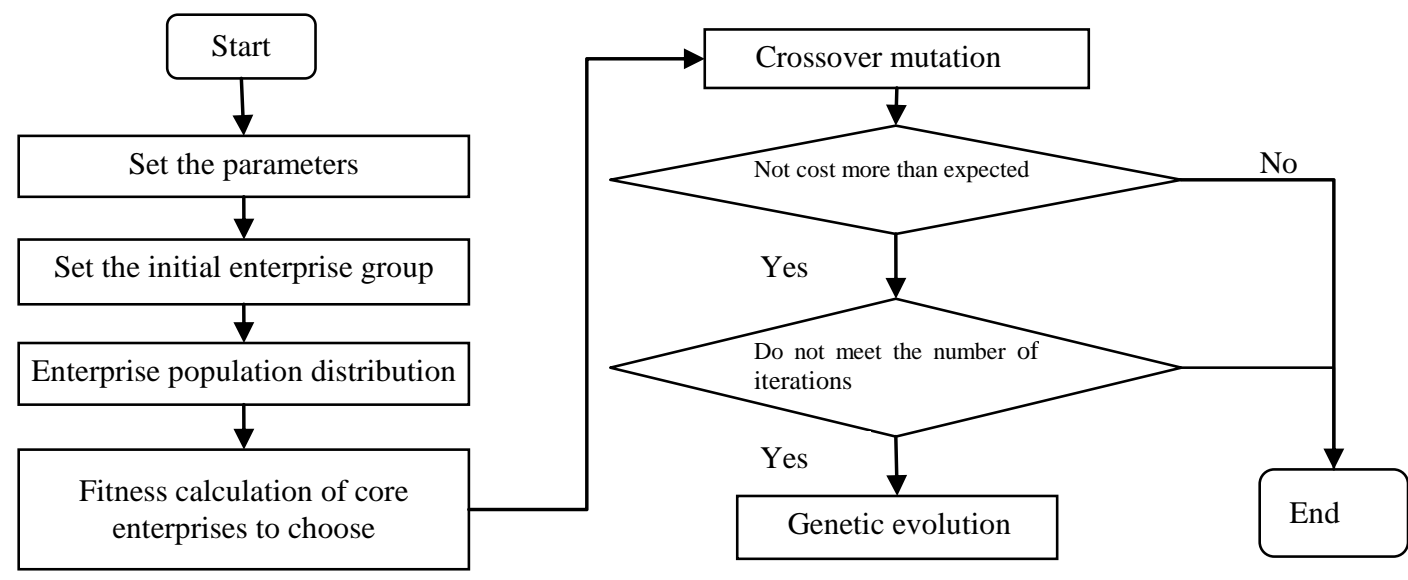

Figure 1 the small micro enterprise niche genetic algorithm based on the cost function

Figure 1 shows a small micro enterprise in multinational management of the development process. Small micro enterprise multinational management is to seek the path of rapid development, study and communication is the important way of growth, micro and small enterprises is relatively sensitive to cost, if the cost surpassed the expectations of the enterprise, small micro enterprise mostly choose to wait, often in a passive state. When companies can pay learning communication cost, and wasn't up to the number of system evolution, the enterprise will produce new fitness, and the new fitness will better than in the past, but also can reduce corporate fitness as there is little probability.
Between the transnational operation of enterprises, considering the multiple constraints study and communication, choose a more feasible study and communication mode, suitable for risk control, security of transnational operation strategy goal. On the basis of the existing multinational management theory, this paper puts forward the following three modes, and cost analysis of patterns.

\subsection{Technology following the innovation model}

In developing countries, multinational business advantage, technology advantage is one of the main advantages, mainly embody in small-scale production technology advantage, technology 
localization, local technical improvement, for the international advanced technology, can be copied by imitation and absorb advanced technology, adjust the production process. This kind of mode of $\mathrm{di} / \mathrm{Dm}$ is relatively small, and the population entropy $\mathrm{H}$ is bigger, so the goal is relatively clear, search cost is low, but resources to swap the demand is higher, may want to try many times, the generally high switching costs. From OEM to independently design, for example, to create brand, by the underlying to regional agents, to the regional agents, micro and small enterprises may be multi-tiered OEM producers of the bottom of society, can indirectly absorb the international advanced production technology and management experience.

\subsection{Sales market integration mode}

In transnational operation, cultural integration is crucial, some scholars put forward the concept of "cultural distance" and "psychological distance", think the geographic, political, and cultural similarity and adjacent similar level of economic development of countries or territories in the market, there are many similarities, it also makes some enterprises have a "natural" competitive advantage, enterprise can through the use of the resources of many countries, sell products to several countries, so as to obtain a competitive advantage. This kind of mode of $d_{i} / D_{m}$ is larger, and the population entropy $\mathrm{H}$ is small, high search costs, and resource exchange cost is low, because this kind of management pattern of the core competence is often focused on a small amount of intangible capital, including market channels, management talent and supply and marketing information, etc. If small micro enterprise take sales market integration mode, and each has different resource of enterprise strategic alliance formation, fusion in the market, to reduce the cost of study and communication.

\subsection{Alternative model of personnel exchanges}

International operator is the core of the development of small micro enterprise multinational management, policies to encourage, capital supplement, technological progress and other factors need to be thought to capture and create. To reduce the small micro enterprise study and communication cost, mining is crucial for talent. International experience is an important decisive factor of enterprise multinational management, and talent is complex, carrying a variety of experience, itself contains information such as market, technology, capital, policy, personnel for the development of the enterprise leading role is critical, and talent distribution between different enterprises. This kind of mode of $\mathrm{d}_{\mathrm{i}} / \mathrm{D}_{\mathrm{m}}$ is opposite bigger, the population entropy $\mathrm{H}$ also is bigger, This kind of pattern search cost is higher, because talent investigation and excavation is a complicated work, resource exchange cost is higher, because the personnel exchanges or replace also need a longer time to reap the benefits, even asked to enterprise itself has the strong ability of change. The appropriate talent flow and matching mechanism is advantageous to the small micro enterprise community resources integration, on the whole reduces the learning communication cost between enterprises, so as to improve the competitiveness of the community.

When the $\mathrm{d}_{\mathrm{i}} / \mathrm{D}_{\mathrm{m}}$ is relatively small, and the population entropy $\mathrm{H}$ is small, also shows that the small micro enterprise in community at the heart of the action, plays a leading role, in small micro enterprise relatively mature period, is entering the scale stage, into the medium and the rapid development of the enterprise, more than the above three kinds of models to choose from.

\section{CONCLUSIONS}

This paper presents a small micro enterprise multinational business niche evolution algorithm, the niche of the algorithm associated with the influence of the small micro enterprise multinational management mechanism, and from the perspective of learning communication cost measure the feasibility of small micro enterprises transnational operation, so as to improve enterprise competitiveness static evaluation method, form a dynamic evaluation method based on global. From multiple factors such as search cost and resource exchange cost considerations, proposed technology with innovation, sales integration, personnel exchange swap three learning communication modes, assist the government and industry improvement of policies and strategies in the enterprises growth path.

\section{REFERENCES}

[1] Lall S, Chen E. The new multinationals: The spread of third world enterprises. Chichester: Wiley, 1983.

[2] Wells L T. The internationalization of firms from the developing countries. Division of Research, Graduate School of Business Administration, Harvard University, 1976.

[3] Ozawa T. International investment and industrial structure: New theoretical implications from the Japanese experience. Oxford Economic Papers, 1979, 31(1): 72-92.

[4] Lin Zhouyu, Lin Hanchuan, Chen Yantai. China's small micro enterprise solution mechanism innovation and the implementation path. Modernization of Management, 2013 (2): 65-67.

[5] Development Research Center of the State Council Enterprise Institute Research Group. Chinese enterprise internationalization strategy. Beijing: people's publishing house, 2006. 
[6] Song Yanqing, Sun Weidong. Small and medium-sized enterprise international market entry mode choice analysis. Science and technology management research, 2013, 33(6): 105-110.

[7] Zhao Chi, Zhou Qin. Small and mid-sized enterprise growth research based on the self-organization perspective. Soft Science, 2011, 25(10): 94-100.
[8] Hua Jie, Cui Duwu. The adaptive niche genetic algorithm based on individual optimization. Computer engineering, 2010, 36(1): 194-196.

[9] Liang Changyong, Cai Meiju, Lu Qing. Improved niche genetic algorithm for hidden target optimization and its application in automobile modeling design. Chinese Journal of Management Science, 2011, 19(1): 126-134. 